\title{
INGRID KÜHN
}

\section{Alltagssprachliche Textsortenstile}

\section{Sprache nach der Wende}

„Unsere Sprache kann man ansehen als eine alte Stadt: Ein Gewinkel von Gäßchen und Plätzen, alten und neuen Häusern, und Häusern mit Zubauten aus verschiedenen Zeiten; und dies umgeben von einer Menge neuer Vororte mit geraden und regelmäBigen Straßen und mit einförmigen Häusern." (Wittgenstein; Philosophische Untersuchungen. $\S 18)$

Bleiben wir bei den geraden und regelmäBigen Straßen - gebaut in den alten Bundesländern ebenso wie in der ehemaligen DDR, und lassen Sie sich in den sprachlichen Alltag der neuen Bundesländer führen. Es gehörte zur politischen Symbolik der DDR, massenhaft und massiv Geschichtszeichen zu setzen.

Straßen, Brücken, Plätze ebenso wie Schulen, Kindergärten, Briefmarken und Fahnen dienten der Selbstdarstellung.

Die Neubaugebiete oder neuen Vororte der ehemaligen DDR trugen Straßennamen, wie Straße der Waffenbrüderschaft, Straße des 90. Jahrestages der DDR, Straße des Roten Oktober oder Straße der Neuerer (Kühn 1992).

Die Bewohner muBten damit leben und protestierten sofort nach der Wende dagegen. Neubenennungen, Rückbenennungen, Umbenennungen erfolgten in den letzten Jahren in den neuen Bundesländern. Neue Namen machen heute die Orientierung schwer für den Bürger in seiner Stadt.

So wie die alten Straßennamen wurden auch mühelos andere hochfrequente DDR-Neuwörter aus dem Sprachgebrauch gestrichen: Parteilehrjahr, Klassenfeind, Kombinat, Subbotnik oder marxistisch-leninistische Weiterbildung.

Dafür dringen neue Wörter ein - und nicht nur am Rande oder als neue Vororte, mehr als Gewerbegebiete. Bestimmte Wortfelder der Alltagssprache sind durch häufigen Gebrauch besonders relevant, und der Orientierungsbedarf in einer fremd empfundenen öffentlichen Kommunikation ist groß. Berufsbezeichnungen sind neu und fremd, entsprechen nicht tradierten Formen, angefangen vom stigmatisierten Arbeitgeber und $A r$ beitnehmer zum Bediensteten, Beamten, Referendar, Akademischen Rat, Azubi, Dezernenten, Ministerialdirigenten bis zum Klientel oder Mittelstand. 
Die Bürger sollen sich angesprochen fühlen und die neue Lexik auch in ihren Anträgen und Bewerbungen verwenden, wie etwa Existenzgründer, Neueinrichter, Nebenverdienstsuchender. Es soll über Teamgeist, Laufbahn, Karriereeinbindung, Referenzen, Führungserfahrungen oder Drittmittel berichtet werden.

Die Reihe läBt sich fortsetzen, und die Auswahl beruht auf Erfahrungen mit der Sprachberatung des Germanistischen Instituts in Halle. Traditionelle Denk- und Verhaltensmuster stimmen nicht mehr, bzw. reichen nicht aus.

Alle Lebensbereiche sind von Veränderungen betroffen. Veränderung erfordert Anpassung. Übernahme von angepaBten wirtschaftlichen, sozialen oder wissenschaftlichen Strukturen erfordert Übernahme von Lexik. Neue Lexik und neue Texte in allen Lebensbereichen müssen schnell verinnerlicht werden - das führt zu Verunsicherungen und Fremdheitsgefühl (Hellmann 1990; Schröder 1992).

In den 40 Jahren der Teilung Deutschlands haben sich in beiden Teilen des Landes unterschiedliche Konventionen auch im kommunikativen Bereich herausgebildet. Ein so umfassender, politisch motivierter Sprachund Kommunikationswandel, wie er sich vollzieht, schlieBt den Textsortenwandel mit ein.

Verständigungsprobleme in verschiedenen Bereichen der Alltagssprache sind verschieden stark ausgeprägt. Es bedarf systematischer Analysen zur Darstellung der Andersartigkeit bestimmter Sprach- und Kommunikationsmuster und parallel dazu der Entwicklung von Hilfeleistungen - vor allem für Ost, aber auch für West - im Umgang mit fremd empfundenen Sprachvorbildern auf allen Gebieten des täglichen Lebens. Die mehrfach beschriebenen Selbst- und Fremdbilder Ost und West zeigen den momentanen Trend: Die „Prestigevarietät” Westdeutsch besetzt den sprachlichen Markt (Resendiz 1992; Lux 1993). Oder anders ausgedrückt: Zur neuen Asymmetrie der Kommunikation gehört, daß der bisherige westdeutsche Sprachgebrauch den Geltungsbereich "gesamtdeutsch" erreicht (Pätzold 1992; v. Polenz 1993). Diskussionen zu Sprachproblemen sind in Tageszeitungen und im Rundfunk aktuell und die Reaktionen in Leserbriefen beweisen die Sensibilität der Betroffenen, wie folgende Beispiele zeigen:

- „Es wundert mich, daß sich jemand darüber wundert, daß die Sprache ausschließlich in Richtung West-Ost geht. Hat man noch nicht gemerkt, daf nicht nur die Sprache, sondern überhaupt alles in Richtung West-Ost läuft? Ich gehöre zu der ausgegrenzten Randgruppe der Vorruheständler und damit zu den Bedauernswerten, denen es nun nicht gelingen wird, dank eines schnellen Lernens des neudeutschen Wortschatzes ein richtiger Mensch zu 
werden. Und dazu muß ich sagen: Ich will nicht. Ich sehe überhaupt nicht ein, warum ich, um bei dem Beispiel zu bleiben, 'cash' sagen muB, wenn es dafür das deutsche Wort 'bar' gibt, was auch hierzulande schließlich jeder versteht."

- „Es ist also verdächtig oder sogar 'out', hier im Osten weiter von der 'Kaufhalle', von 'viertel acht' oder vom 'Kollektiv' zu reden. Ich möchte statt der im MZ-Beitrag angeführten Belanglosigkeiten dieses in meinen Augen Sprachterrors einmal ein paar wesentliche neue Vokabeln nennen, die hier hereingebrandet sind: Massenarbeitslosigkeit, Ministergeldskandale, abwickeln, feuern, Obdachlose, Aussteiger, Reps, Rauschgiftszene, Kinderfeindlichkeit, gegauckt werden."

(Mitteldeutsche Zeitung, 26.2.1994)

Momentan ist in der Alltagssprache der neuen Länder eine Mischung von tradierten Wendungen im neuen Diskurs zu beobachten, d.h., alte kommunikative Verhaltensweisen werden auf strukturell vergleichbare modifiziert übertragen. Der gemischte Sprachgebrauch ist gruppenspezifisch und betrift unterschiedliche Textsorten.

Neue Führungskräfte werden schon berufsbedingt sehr schnell situationsspezifische Sprachregister ziehen, neue Muster übernehmen und aktiv und passiv verwenden. Trotzdem muB auch bei ihnen damit gerechnet werden, daB in selbstverständlicher UnbewuBtheit kognitive Muster und ihre sprachliche Umsetzung bewahrt, variiert und differenziert werden, was wiederum bei Kommunikationspartnern aus dem Kommunikationsgebiet „Westdeutsch” je nach Toleranzspielraum, Verwunderung, Fremdheit oder Überheblichkeit auslöst. So gab es eine erregte Leserbriefdiskussion um das Neuwort (DDR) Dispatcher, das ein westdeutscher Unternehmer hier in dieser breiten Verwendungsweise ungewöhnlich fand. Die Reaktion "Ost" zeigt nochmals die momentane Sprachsituation.

„Dispatcher-Unsinn?
Daß wir aus 'Neufünfland' für manche Bürger jenseits der Elbe im all-
gemeinen viel Nachholbedarf haben, ist ja bekannt. Aber daß wir nun
noch der deutschen Sprache unkundig sind und wenig Allgemeinwissen
besitzen und noch Unsinn reden, das haut den stärksten Eskimo vom
Schlitten. Fast jeder weiB, was das Wort Dispatcher bedeutet. Dispat-
cher zum Beispiel ist echt ein Arbeits- und Verkehrskoordinator. Wenn
Herr Overhues bestimmte Wörter nur aus einem Metier kennt, kann
man ihm nur raten, seinen Horizont zu erweitern. Havag-Dispatcher
sind jedenfalls meist Könner ihres Faches." (Mitteldeutsche Zeitung, 2.3.1994)

Andererseits erfolgt zur Zeit ein Funktionswandel DDR-typischer Bezeichnungen oder typischer Wortgruppen, die als alltagsweltliche Wortschätze tief habitualisiert sind, auch wenn sie in bestimmte neue Textmuster eingefügt werden. (Wissenschaftler: Begründung C4-Berufung entspricht den gesellschaflichen Erfordernissen; Arzt: die medizinische Ver- 
sorgung im Territorium; Verkäuferin: Ich wollte in meiner Verkaufsstelle bleiben; Studentin: Wissenschaftliche Hausarbeit - Staatsexamensarbeit; Journalist: Er übernimmt in einem Sanatorium eine leitende Funktion; Betrieb annonciert: In unserem Ferienobjekt sind freie Ferienplätze.)

Bei diesem synonymen Gebrauch von vertrauter Lexik und von neuen Benennungen für gleiche Sachverhalte steht die Frage nach Präferenzen und doch möglichen Bedeutungsdifferenzen. Das temporäre $\mathrm{Ne}$ beneinander gruppenspezifisch unterschiedlichen Sprachgebrauchs bringt zunächst Mischung im ProzeB der Angleichung kommunikativer Verhaltensmuster. Gegenseitige Kenntnisnahme synonymer Lexik als Wortdubletten im Sinne von arealer Spezifik ist für Kommunikationspartner aus Ost und West wichtig (Lerchner 1992).

BewuBt vermieden werden Kaderentwicklung, Kaderakte, obwohl unbewuBte Mischungen zu Stellenanzeigen mit folgender Benennung führen: geeigneten $K$ a de $r$ mit Wohnsitz in Leuna, gesucht ...

Formen von Adaption und Loyalität führen zu stilistischen Veränderungen bei bestimmten Textsorten. Solche Veränderungen sollen am Textmuster "Arbeitszeugnis/Beurteilung" verglichen werden mit konventionell vorgegebenen Rahmen, die sich momentan - wie in diesen einleitenden Bemerkungen angedeutet werden sollte - in den neuen Bundesländern gravierend verändern und bei dieser Textsorte konfliktreich verlaufen. Die hier darzustellenden Stilveränderungen resultieren aus meiner Auffassung von Stil als einem Textphänomen, als einem Teilaspekt des Textes in einer strukturellen Mehrstufigkeit (Sandig 1986, S. 128), wo eine Reihe von Strukturelementen stilistische Funktionen haben.

Hier wird besonders die lexikalische Teilebene untersucht, wobei lexikalische Besonderheiten als Index für Veränderungen im Vergleich stehen und einen besonderen Stilwert bekommen. Die Veränderung stilistischer Durchführungsbestimmungen sind der veränderten sozialen Situation geschuldet. So ist Stil auch zu sehen als ein Mittel der Anpassung sprachlicher Handlungen an Situationen.

Die Konflikthaftigkeit der Stilwirkung kann nur partiell benannt werden und resultiert mit aus den aufzuzeigenden stilistischen Veränderungen, die in der Relation von der Handlungsdurchführung zur Situation und den veränderten sprachlichen Mitteln zu sehen ist. So stehen das Erwartbare und Angemessene - weil bisher konventionell Übliche - den Besonderheiten von neuer Angemessenheit und normativen Vorgaben in einer Mischung gegenüber. Veränderungen der Textstruktur führen zII Veränderungen des Stils - mithin zu Stilwandel. 


\section{Aspekte bei der Beschreibung des Textmusters Arbeitozeugnis/Beurteilung}

\subsection{Alltagssprachliche Textsorten}

Ohne die verschiedenen und vielfach beschriebenen Textsortenklassifikationen zu referieren - die letzte ausführliche Übersicht dazu bietet meines Wissens Rolf (1993) „Die Funktion der Gebrauchstextsorten” sollen hier momentane Veränderungen in der Orientierung von Kommunikationsteilnehmern in der kommunikativen Praxis an Einzelbeispielen benannt werden.

Auf der Grundlage einer funktionalen, pragmatischen Textklassifikation wird von der Textfunktion als einem kommunikativen, handlungsbezogenen Kriterium ausgegangen. Dabei wird die Textfunktion als Typologisierungsbasis für Textklassifikationen verstanden (vgl. auch Brinker 1985; Franke 1987; Gläser 1990).

Textsorten werden aufgefaBt als konventionalisierte Muster für komplexe sprachliche Handlungen. Beschreibbar sind sie auf der Basis ihrer Funktion, ihrer kontextuellen/situativen Bedingungen und ihrer sprachlichen Merkmale. Alltagssprachliche Textsorten gehören zum Alltagswissen, wobei die lexikalisierten Textsortenbezeichnungen häufig der Textfunktionsbezeichnung dienen. Die Textsortenbezeichnung kann als „Präsignal” (Große 1976, S. 20) oder als „explizit performative Formel” (Rolf 1993, S. 146) betrachtet werden und ist als Indikator für die Textfunktion zu berücksichtigen.

Alltagssprachliche Textsorten sind zweckbestimmt und somit pragmatisch orientiert, dazu gehören auch bestimmte Fachtextsorten, die für das Alltagsleben bestimmt sind und von Bürgern in einer bestimmten institutionellen Hierarchie verfaBt werden. Konventioneller Stil und individueller Spielraum sind differenziert ausgeprägt in den verschiedenen Textsorten. Alltagssprachliche Textsorten sind differenziert normiert. Die Normierungskriterien sind hinlänglich bekannt und ihre sprachliche Gestaltung ist je nach Normierungsgrad vorgeprägt. Die Textsorte bildet also einen normierenden Orientierungsrahmen, häufig mit Anleitungen zum Formulieren (Gülich 1985, S. 38/39).

Alltagssprachliche Textsorten sind in der Sprachgemeinschaft historisch gewachsen. Typische Muster sind intersubjektiv verfestigt.

Der gemeinschaftliche Wissensvorrat unterliegt Veränderungen, wenn sich konstitutive Bedingungen, die textextern sind, verändern. Dazu gehört die Kommunikationssituation als eine Form der situativen Beschreibungsebene. Spezielle Handlungs- und Bewertungsnormen gel- 
ten für bestimmte gesellschaftliche Bereiche. Feste Redekonstellationen führen zu festen Sprachhandlungsmustern.

Wie situative Veränderungen zu Veränderungen verschiedener Textsortenstile führen, kann vorläufig nur an einzelnen Textexemplaren gezeigt werden. Fixierte Situationsbedingungen sowie Kenntnis aller Sprachteilhaber über diese Bedingungen führten zu ritualisierten Textmustern. Man kann von einem „Erzeugungszusammenhang” (Franke 1991, S. 168) zwischen der situativen und sprachlichen Ebene sprechen. Situative Bedingungen bilden hier den Hintergrund bzw. die Ursache für die Entstehung von Musterwandel.

Das Wissen davon, daß die kommunikativen Traditionen so nicht weiterexistieren, die partielle Inkompatibilität alltagssprachlicher Textsorten, führt gegenwärtig zu einer Mischung aus Bewahren und Adaptieren von Wissensmustern, realisiert in alltagssprachlichen Textsorten mit gemischten sprachlichen Mitteln aus altem und neuem Diskurs. Anders gesagt - zwischen altem Textsortenwissen und der momentanen Realisierung im Text entstehen Musterkonflikte, die zu Konfliktsituationen in der Kommunikation führen.

Textsorten wie Leserbrief, Kunstkritik, Bewerbungen oder Stellenanzeigen in den Tageszeitungen zeigen diese Veränderungen deutlich.

\subsection{Beurteilung und Arbeitszeugnis - zwei Namen ein Konzept?}

Bei einer Textsortendifferenzierung kann man von personenbezogenen Textsorten sprechen, bei denen neben einer Bescheinigung oder Bestätigung von Tatsachen auch eine Bewertung erfolgt. Eine Bewertung, die Auskunft über eine Person gibt, da eigene Personenkenntnis nicht vorhanden ist. Rolf zählt Texte dieser Art zu "Leistungs-/Eignungsbezogene Textsorten" (Rolf 1993, S. 302). Neben Arbeitszeugnis stehen Dienstzeugnis, Abiturzeugnis, Befähigungsnachweis u.a.m.

Wichtiges Kriterium ist zusätzlich zum Bescheinigen das Bewerten (Impfzeugnis/Arbeitszeugnis). Die Auszählung von Dimter 1981 (S. 33) zu alltagssprachlichen Textklassennamen brachte über $1500 \mathrm{im}$ Duden benannte Namen von Textklassen, von denen 480 für grundlegende Textklassenkonzepte stehen, zu dem auch „Zeugnis” gehört.

Welche alltagsweltlichen Konzepte liegen den konventionellen sprachlichen Ausdrücken Beurteilung und Arbeitszeugnis zugrunde?

Jeder ehemaliger DDR-Bürger kennt Beurteilungen, jeder hat mehrfach Einschätzungen seiner Leistungen gelesen, meist schöngefärbt gefunden, dienten sie doch hauptsächlich zur Prämierung zum 1. Mai, zum Inter- 
nationalen Frauentag, Tag des Gesundheitswesens, Tag des Lehrers, Tag des Bauarbeiters oder zur Aktivistenauszeichnung, zur Auszeichnung als Kollektiv der sozialistischen Arbeit. Aber auch finanzielle Höherstufungen muBten begründet werden, dienstlicher Aufstieg war in Kaderperspektivplänen fixiert und verlangte umfangreiche Beurteilungen, die als "exklusive Kommunikation" dem Beurteilten unbekannt waren (Fix 1993). Ansonsten hat jeder Abteilungsleiter mit oder ohne Hilfe des Arbeitskollektivs, der Gewerkschafts- und Parteileitung Beurteilungen geschrieben.

Ziemlich schnell - im zweiten Jahr nach der Wende etwa - verlangten die ersten Mitarbeiter, die sowohl durch Kündigung oder besser durch Aufhebungsvertrag den Betrieb, die Firma verlassen mußten, ein Arbeitszeugnis. Die Leiter - alte oder neue - aber aus der ehemaligen DDR, hörten den Terminus erstmals, hatten aber zunächst keine Probleme mit der inhaltlichen Zuordnung. Beurteilung als Überschrift blieb meist stehen.

Im Duden (Ost) oder Wörterbuch der deutschen Gegenwartssprache nachgeschlagen, findet man Arbeitsnorm, Arbeitsplan, Arbeitsproduktivität, Arbeits- und Lebensbedingungen und Arbeitszeit. Arbeitszeugnis erscheint nicht. Im Synonymwörterbuch bei Beurteilung zur Vergewisserung nachgesehen, stehen als Synonyme:

Begutachtung, Bewertung, Wertung, Einschätzung, Urteil, Charakteristik.

nicht aber Arbeitszeugnis.

Es ist festzustellen, daß es die Benennung Arbeitszeugnis in der DDR nicht gab, während sie in den alten Bundesländern seit Anfang der 50er Jahre üblich wurde.

Wenn ein Kriterium für ein Textmuster die Benennung und damit Alltagskategorisierung ist, so werden hier als musterbezeichnende Ausdrücke Beurteilung und Arbeitszeugnis synonym verwendet. Strukturell vergleichbare Kommunikationsmuster und entsprechendes Musterwissen müssen zu Abwandlungen bei der Realisierung des Textmusters führen. Intersubjektive Wissensbestände sind unter den veränderten situativen Bedingungen modifiziert $2 u$ realisieren. Solche Veränderungen verlangen zunächst Musterkenntnis. 


\section{Textmusterbeschreibung des Textmusters Arbeitszeugnis/Beurteilung}

\subsection{Norm und Aufbau von Arbeitszeugnissen}

Wichtigster Grund für das Verfassen von Arbeitszeugnissen ist der Arbeitsplatzwechsel. Das Bürgerliche Gesetzbuch benennt die allgemeine Rechtsgrundlage:

„Bei der Beendigung eines dauernden Dienstverhältnisses kann der Verpflichtete (Arbeitnehmer) von dem anderen Teil (Arbeitgeber) ein schriftliches Zeugnis über das Dienstverhältnis und dessen Dauer fordern. Das Zeugnis ist auf Verlangen auf die Leistungen und die Führung im Dienst zu erstrecken.” (BGB 630)

Im Duden (1989) steht unter Arbeitszeugnis:

„dem Arbeitnehmer vom Arbeitgeber über Art und Dauer der Beschāftigung sowie über Führung und Leistungen ausgestelltes schriftliches Zeugnis."

Art des Dienstverhältnisses, Dauer sowie Leistung und Führung im Dienst beschreiben die Textfunktionen des Arbeitszeugnisses. Oberster Grundsatz oder Norm der Einschätzung ist die Zeugniswahrheit. Es darf die Bewertung "nicht wesentlich vom Rahmen der Verkehrssitte abweichen, d.h. von dem, was allgemein üblich ist." (Presch 1991, S. 7)

Ratgeberliteratur für das Schreiben und Lesen von Arbeitszeugnissen ermöglicht allen Sprachteilhabern (Produzenten und Rezipienten von Arbeitszeugnissen), von Bedeutung, Art, Formulierung über Geheimcodes bis zu Rechtsfragen das Wissen über Arbeitszeugnisse zu vervollkommen (Dittrich 1988; Wypijeski 1992; Nasemann 1992; Ruge 1993).

Die verschiedenen Arten von Arbeitszeugnissen werden in der Ratgeberliteratur ausführlich beschrieben. Wichtigste formale Differenzierung ist die $Z$ weiteilung in einfache und qualifizierte Arbeitszeugnisse. Daneben erfolgt noch die Beschreibung von Zwischenzeugnissen, Ausbildungszeugnissen, Probezeugnissen u.a.m. (Dittrich 1988, S. 44).

Das qualifizierte Arbeitszeugnis hat folgenden Aufbau (Presch 1991, S. 2):

1. Angaben zur Person, Beschäftigungsdauer

2. Tätigkeitsbeschreibung

3. Leistungsbeurteilung

4. Führung/Sozialverhalten

5. Führungsqualitäten/Aufstiegseignung

6 . Kündigungsgrund

7. SchluB (Wünsche/Unterschrift). 


\subsection{Norm und Aufbau von Beurteilungen}

Ratgeberliteratur zum Schreiben von Beurteilungen gab es in der DDR nicht. Dafür wurden in sprachwissenschaftlichen Fachbüchern die Anforderungen an Beurteilungen benannt und erläutert. Sie wurden auch in den Berufs-, Ingenieur- und Fachschulen im Fach "Deutsch" unter dem Terminus "Gebrauchsformen des Sachstils" neben dem „Wirtschaftsbrief”, „Aussprache zwischen Bürgern und staatlichen Stellen”, „Bewerbungsschreiben” und "Kadergespräch” geübt (Ausdruckslehre 1969). Für das Schreiben von Schülerbeurteilungen gab es ein Anleitungsbuch, in dem das Ziel der Schülerbeurteilungen einleitend beschrieben wird, und übergreifend für alle Beurteilungen gilt:

„In der sozialistischen Gesellschaft sind kontinuierliche Persönlichkeitsbeurteilungen und Analysen des Kollektivs ein unerläßlicher Bestandteil der wissensch aftlichen Leitungstätigkeit auf verschiedenen Ebenen, und schlieBen eine ständige Analyse und Beurteilung der Persönlichkeit und ihrer Entwicklungsbedingungen ein ... Die Entwicklung muB bewuft und systematisch gelenkt werden. ...” (Erlebach 1983, S. 13).

In der Anleitung zur Ausbildung von Deutschlehrern heißt es:

„Beurteilen beruht auf Bewerten ... Das Bewerten erfolgt auf der Basis von Parteilichkeit, in unserem Falle von sozialistischer Parteilichkeit ..." (Schmidt 1981, S. 159).

In dem Buch „Deutsche Sprache - Handbuch für den Sprachgebrauch” steht:

„Bei Beurteilungen haben wir die Aufgabe, einen Kollegen nach den Forderungen, die wir an eine sozialistische Persönlichkeit stellen, einzuschätzen ... Wir gehen deshalb bei der Beurteilung eines Kollegen von seiner Beziehung zur sozialistischen Gesellschaft aus ..." (Liebsch 1976, S. 437).

Aufbau einer Beurteilung (Liebsch 1976, S. 438)

1. Überschrift

2. Personalangaben

3. Tätigkeit im Betrieb

4. Berufliche Entwicklung

5. Einstellung zur Gesellschaft

6. Einstellung zur eigenen Person

7. Beurteilung besonderer Eigenschaften

8. Zweck der Beurteilung

9. SchluB 
3.3 Dominierende Illokutionen und Handlungshierarchie des Textmusters Arbeitszeugnis/Beurteilung

Als (deklarative) Textsorten mit Leistungs- und Verhaltensbewertung ordnen sich Arbeitszeugnisse und Beurteilungen in das vorgegebene Wertgefüge ein, sind wertende Stellungnahmen auf der Basis der fixierten Normen mit prototypischen Formulierungen im Textmuster. Das gilt so allgemein für beide Varianten des Textmusters, nur daß die Wertmaßstäbe unterschiedlich waren.

Zum Arbeitszeugnis sind konventionelle Vorgaben und Formulierungsmuster in jedem Ratgeber beschrieben, beispielsweise in einer sechsstufigen Leistungsbewertung, in Bausteinen, die Verhalten, Verantwortungsbereitschaft, Vertrauenswürdigkeit oder Führungsqualitäten und Kreativität beschreiben. Gleich dazu werden "Lesehilfen" zur Interpretation vorliegender Zeugnisse gegeben (Dittrich 1989, S. 74ff.).

Wie Norm und Aufbau als Gliederungskriterien zeigen, besteht das $\mathrm{Ar}$ beitszeugnis aus mehreren obligatorischen Teiltexten, die hauptsächlich eine Tätigkeitsbeschreibung, Bewertung von Leistung und Beurteilung von Sozialverhalten geben. In den Teiltexten 1. (Angaben zur Person), 2. (Tätigkeitsbeschreibung) dominiert die Informationsfunktion mit den illokutiven Teilhandlungen Feststellen, Beschreiben, Begründen, Zusammenfassen. Daraus resultiert eine orientierende Funktion für einen neuen Arbeitgeber.

Bewertungen erfolgen in den Teiltexten 3. (Leistungsbeurteilung), 4. (Führung/Sozialverhalten), 5. (Führungsqualitäten/Aufstiegseignung).

Sie werden meist mit Hilfe der vorgegebenen Werteskala ebenso wie in der Bewertung des Sozialverhaltens durch intertextuell fixierte sprachliche Muster realisiert. Die Bewertung der Leistung soll wohlwollend sein und dem Fortkommen dienen.

Die dominierenden Illokutionen, die auch schon in der Gesetzes- und Dudenbeschreibung als Indikatoren für eine Handlungshierarchie gegeben sind, lauten:

Das Beschreiben und Bewerten

- soll dem Beurteilten zum Fortkommen dienen,

- soll dem Beurteilten nicht schaden (Wohlwollen),

- soll zuverlässig unterrichten,

- soll Auslese ermöglichen. 
Als obligatorische Teiltexte für Beurteilungen gelten die bei Norm und Aufbau beschriebenen Gliederungsanweisungen.

Die Teiltexte 1. (Angaben zur Person), 2. (Tätigkeitsbeschreibung und vorherige Tätigkeit) informieren, wobei durch Angaben zur vorherigen Tätigkeit/Entwicklung bereits inklusive, indirekte Einschätzungen erfolgen.

Beispiel:

„Dr. X, geb. 1957, hat nach dem Abitur mit Auszeichnung ein Vollstudium in der Sowjetunion in Rostow am Don absolviert. Auf Grund seiner Studienleistungen (Diplom mit Auszeichnung 1981) und Auszeichnung als Jungaktivist wurde ihm eine Aspirantur in Rostow vorgeschlagen, die er 1984 mit der Verteidigung seiner Kandidatendissertation abschloß. Im Herbst $1984 \mathrm{nahm}$ er seine Tätigkeit auf. Er ist seit 1985 unbefristeter Assistent."

Die Teiltexte 3. (Berufliche Entwicklung/Einstellung zur Arbeit und zum Kollektiv), 4. (Einstellung zur Gesellschaft), 5. (Einstellung zur eigenen Person) haben argumentierenden und bewertenden Charakter.

Beispiele:

(1) „Sein überaus positiver Einfluß auf die Studenten und Führungskader der II. Abteilung war gekennzeichnet von hoher Disziplin, Parteilichkeit und großer Fachkenntnis. In seiner sehr interessant gestalteten politischideologischen Arbeit genoB Gen. Dr. X bei allen hohes Ansehen."

(2) „Dr. X ist verheiratet, hat zwei Kinder im Vorschulalter und lebt in geordneten Familienverhältnissen. Persōnliche Kontakte zu Verwandten im NSW bestehen nicht, gelegentliche wissenschaftliche Kontakte zu Slawisten aus NSW-Ländern dienen der notwendigen wissenschaftlichen Kommunikation."

Die Teilhandlung „Beurteilen” kann als wesentlich gelten. Es kommen aber implizit und explizit noch die Zusatzhandlungen "Erziehen” und „Prognostizieren” (nützliches Mitglied unserer Gesellschaft/potentiell entwicklungsfähig) hinzu (vgl. dazu ausführlich Fix 1993).

Beispiele:

(1) „Nach alldem sind wir der Gewißheit, daß wir in Genossin Dr. $X$ eine sozialistische Hochschullehrerpersōnlichkeit besitzen, die ihre gesamte Kraft für Ausbildung und Erziehung sowohl der Studenten als auch des wissenschaftlichen Nachwuchses einsetzt und die in hohem Maße dazu beiträgt, die gesellschaftliche Wirksamkeit ihres Faches weiter auszuprägen."

(2) „Die Parteigruppe unterstützt und begrüBt die Entscheidung der SL und PL der Kollegin X eine Oberassistentenstelle zu geben und erhofft sich ein stãrkeres Engagement und eine größere Wirksamkeit der gesellschaftspolitischen Arbeit der Kollegin X."

(3) „Es wäre wünschenswert, wenn Kollegin X sich zur Vollschwester qualifizieren würde." 
Obligatorisch ist auch der Teiltext 6. (Zweck der Beurteilung), da die vielfältigsten Gründe für das Schreiben einer Beurteilung vorlagen.

Beispiele:

(1) Aktivist der sozialistischen Arbeit: „Seit November 1978 leistet Genos$\sin \mathrm{X}$ eine verantwortliche Arbeit in der Parteileitung der GO, setzt sich mit Engagement und Selbstlosigkeit für die Verwirklichung der Parteibeschlüsse ein und wird mit Nachdruck ihrer Aufgabe bei der Kassierung der Parteibeiträge gerecht."

(2) Höherstufung: „Die Einstufung von Kolln. Dr. X muß von der Parteileitung vorgenommen werden, da ihre erziehungswirksamen Aktivitäten und ihre groBe Belastung vor allem durch ihre politischen Funktionen zustande kommen."

Die intertextuelle Erfahrung mit dem Textmuster, die jeder arbeitende DDR-Bürger als Beurteilter gemacht hat, lautet: Fachliche und gesellschaftliche Aktivitäten müssen umfassend beschrieben (schöngefärbt) werden. Beurteilungen sind häufig zwei bis vier Seiten lang. Der zweiseitigen Zweckbestimmung von Arbeitszeugnissen (Fortkommen und Auslese) entspricht eine Mehrdimensionalität von Beurteilungen:

- Information bei Arbeitsplatzwechsel,

- Leistungseinschätzung,

- Einschätzung des Entwicklungsstandes des Werktätigen,

- Mittel der Erziehung und Kritik.

\subsection{Formulierungshilfen für Sequenzmuster im Vergleich}

Entsprechend dem Prinzip der logischen Abfolge sind bei dieser stark normierten Textsorte zu gesellschaftlichen Standardzwecken Sequenzmuster als globale Vorgaben vorhanden. Formulierungshilfen werden als textmusterspezifische Ausdrücke der einzelnen Sequenzen gegeben.

Die Hilfen zum Formulieren von Arbeitszeugnissen sind perfektioniert und bestehen aus einer Zusammenstellung von „Formulierungsbausteinen", die vor allem arbeitsrechtliche Auseinandersetzungen ersparen sollen (Dittrich 1988, S. 66).

Für Beurteilungen sind in den Lehrbüchern Formulierungen genannt, die hier durch auf Häufigkeitsuntersuchungen basierende Standardformulierungen ergänzt werden. Beispielhaft sollen typische Formulierungsangebote für die einzelnen Sequenzen verglichen werden. Denen lagen ergänzend zu der Ratgeberliteratur und den genannten DDRLehrbüchern 12 Arbeitszeugnisse aus den alten Bundesländern und 85 Beurteilungen aus der DDR-Zeit zugrunde. 


\section{Arbeitszeugnis}

1. Angaben zur Arbeitszeugnis-Person

Anrede: Herr, Frau, auf Wunsch Fräulein

\section{Tätigkeitsbeschreibung}

Bezeichnung von Haupt- und Nebentätigkeiten: „Zu den Aufgaben gehörten ..." "Er leitete die Abteilung ..." (Dittrich 1988, S. 70)

\section{Leistungsbeurteilung}

Skala zur sechsstufigen

Leistungsbewertung

(Dittrich 1989, S. 74)

Note/Bedeutung/Wortbeurteilung (z.B. in Berufzeugnissen):

1 = sehr gut: ... erfüllte stets seine Aufgaben zu unserer vollsten Zufriedenheit ...

2 = gut: ... erfüllte seine Aufgaben zu unserer vollen Zufriedenheit ...

3 = befriedigend: ... erfüllte seine Aufgaben zu unserer Zufriedenheit ...

4 = ausreichend: ... bemühte sich stets, seine Aufgaben zu erfüllen ...

5 = mangelhaft: ... versuchte, den Anforderungen gerecht zu werden ...

\section{Beurteilung}

1. Angaben zur Person

Kollege, Genosse, Jugendfreund

\section{Tätigkeitsbeschreibung}

Neben jetziger Tätigkeit Mitteilung

über vorherige Tätigkeit, z.B.: nach dreijähriger Armeezeit, Studium - vorfristig mit

Diplom abgeschlossen;

3. Berufiche Entwicklung/Einstellung zur Arbeit und zum Kollektiv

- Vorlesung und Seminare zeichnen sich durch immanenten marxistischleninistischen Gehalt aus;

- trägt wesentlich zur gesellschaftspolitischen Erziehung der Studenten bei; - ist ein wertvolles und vielseitig einsetzbares Mitglied des Lehrerkollektivs; Lexeme: verantwortungsbewubt, große Verdienste erwarb er sich, wissenschaftlich fundiert; internationale Anerkennung erworben, vielfältige gesellsch aftliche Arbeit, hohen politisch-erzieherischen EinfluB, Heranbildung politisch bewußter Nachwuchskräfte. 
6 = ungenūgend: ... mühte sich, zu einem Ergebnis zu kommen ...; oder: ... wir wünschen ihm eine Position, die seinen Fāhigkeiten gerecht wird ... (was natürlich sarkastisch gemeint ist!)

\section{Führung/Sozialverhalten} "Stellte stets das Interesse der Firma in den Vordergrund ..." "arbeitet zum Wohle des Unternehmens”, „war für diese Position geeignet", „kollegiale Haltung, Diskretion, Verhalten gegenüber den Vorgesetzten ..." „Kreativität und innovative Fähigkeiten"

(Dittrich 1989, S. 79)
4. Einstellung zur Gesellschaft/ Angaben zur politisch-ideologischen Haltung Zugehörigkeit zu politischen Organisationen: Besucht die Kreisparteischule; leitet ein Kollektiv der sozialistischen Arbeit; Mitglied der SED; Sekretăr der Grundorganisation; als prinzipienfester und verantwortungsvoller Kommunist bewährt; weltanschaulich gefestigtes Engagement; nimmt aktiven parteilichen Anteil an der Kulturpolitik der DDR; Tätigkeit in der sozialistische Presse; als BGL-Vorsitzender hat er sich stets offen zur Politik unserer Partei bekannt; als ein Arbeiterkader hat er ein positives Verhăltnis zur Partei; prägt spürbar die FDJ-Arbeit; nimmt aktiv an der Gestaltung des sozialistischen Wettbewerbs teil; hat einen klaren Klassenstandpunkt - erkennbar durch die fundierte Anwendung theoretischer Kenntnis auf dem Gebiet des M/L bei brennenden Zeitfragen und in der Ũbernahme eines Jugendobjektes; vertritt jederzeit die Position des sozialistischen Staates. 
5. Führungsqualitäten/Aufstiegseignung

„Verhalten bei der Führung seiner Mitarbeiter;

Leistungsfähigkeit unserers

Unternehmens steigern; versteht es zu motivieren; natürliche Autorität;

Führungsfāhigkeit"

(Dittrich 1988, S. 82)
5. Einstellung zur eigenen Person (Leiterpersönlichkeit) Prinzipienfestigkeit, Gründlichkeit, beharrlicher Arbeitswille, genießt hohes Ansehen; Engagement: fand ungeteilte Anerkennung; prägt durch seine Persōnlichkeit die Arbeit; gibt wichtige Orientierungen für die Arbeit; verantwortliche Funktionen; überdurchschnittliche Arbeitsbelastung; größtmögliche Effektivität; sozialistische Hochschulpersönlichkeit; starke Vorbildwirkung; langjährige Leitungstätigkeit; Leiter eines sozialistischen Kollektivs; verantwortungsbewußte Kaderpolitik; Initiative und Tatkraft.

\section{Zweck der Beurteilung} Hōherstufung, Prāmierung; Berufung, Kurantrag, Arbeitsplatzwechsel.

\section{Schluß (Unterschrift}

evtl. Wünsche)

Direktor für Erziehung und

Ausbildung; Sektionsdirektor;

Parteisekreär; BGL-Vor-

sitzender; Chefarzt; Stationsarzt;

AGL-Vorsitzender;

Beurteiler.

(Dittrich 1988, S. 69)

Unterschrift:

Geschäftsfūhrer

Diese Gegenüberstellung zeigt zunächst eine relative Übereinstimmung in den konventionellen Vorgaben, die das Muster bestimmen, was die intuitive Musterkenntnis von Arbeitszeugnis für Beurteilung rechtfertigt.

Die Realisierung unter den veränderten situativen Bedingungen erfordert eine stark modifizierende Ausfüllung des Textmusters. Reicht nun das konventionell unterstellte Musterwissen, das gruppenspezifisch inhomogen ist, aus, um Texte neu-mustergemäß zu formulieren? 


\section{Wissensmuster und ihre sprachliche Realisierung}

Bei der Analyse von Arbeitszeugnissen - geschrieben in den neuen Bundesländern von neuen Vorgesetzten, aber ausschlieBlich ehemaligen DDR-Bürgern - ging es darum, Textmusterrealisierungen $z \mathrm{u}$ beschreiben. Dabei handelt es sich einmal um Analysen von Beurteilungen/Arbeitszeugnissen für Ärzte im Praktikum. Für diese Gruppe standen auch Vergleichsarbeitszeugnisse aus den alten Bundesländern und Beurteilungen von vor der Wende zur Verfügung. Des weiteren wurden Arbeitszeugnisse aus Betrieben und von Universitätsmitarbeitern analysiert. Hinzu kamen noch Beurteilungen bzw. Schullaufbahnempfehlungen aus einzelnen Schulen.

Insgesamt konnten 35 nach der Wende geschriebene Arbeitszeugnisse analysiert werden, so daB hier nur erste Tendenzen anhand des zugrundeliegenden Materials aufgezeigt werden können.

Zunächst wurde jeder Text nach dem Muster in sechs Teiltexten analysiert. Dabei wurde auf die Analysenkenntnisse aus den vergleichenden Untersuchungen von Arbeitszeugnissen und Beurteilungen zurückgegriffen. In einer Tabelle wurden rezeptiv auffällige Formulierungen mit der folgenden Fragestellung eingetragen:

- In welchen Teiltexten gibt es Präferenzen für vertraute Formulierungsmuster?

- In welchen Teiltexten erfolgt selektiv die Übernahme von neuem sprachlichem Inventar?

- Wo wird (ungewollt) individuell formuliert, weil das Textmusterwissen nicht ausreicht?

- Welche Teiltexte wurden eliminiert?

Aufgrund der veränderten situativen Bedingungen und der mehr oder weniger globalen Kenntnis von Musterwissen stehen neue und alte Formulierungsmuster als Synthese für zu dokumentierenden Sprachwandel, der aus einer gesellschaftlichen/soziolinguistischen Drucksituation, verbunden mit außerlinguistischen Begleitumständen, resultiert. Muster befolgen und Improvisieren führen zumindest zu einer vorübergehenden Veränderung des Textmusters bei einer Mischung von Musterwissen.

\subsection{Textmusterbestimmende Typika aus dem alten Diskurs}

Der Begriff Typikum soll vor allem einen Unterschied in der Vorkommensweise, d.h. Seltenheit, eventuelle Beschränktheit auf bestimmte Textsorten implizieren und ist durch die Vorkommenshäufigkeit charakterisiert (Anderson 1984). 
In welchen Teiltexten von Arbeitszeugnissen/Beurteilungen werden alte Muster bewahrt?

Schon die Überschrift wird von einem GroBteil noch mit „Beurteilung” (auch "AbschluBbeurteilung"/ "Gesamtbeurteilung”) angegeben. Im Teiltext 1. Angaben zur Person/Anrede wird deutlich, daß hier fast ausschlieflich DDR-typische Anredeformen übernommen werden: $\mathrm{Kol}$ lege/Kollegin; ohne Titel; ohne Anrede nur Vor- u. Familienname.

Des weiteren fällt auf, daß weibliche Personen sehr häufig mit maskulinen Formen bezeichnet werden, wie etwa:

sie war Lehrer im Hochschuldienst

sie wurde rasch zu einem wertvollen Mitarbeiter im Ärztekollektiv

Frau $X$ arbeitete als Sachbearbeiter in der Vertriebsabteilung.

Die Differenzierung in weibliche und männliche Anrede- und Berufsbezeichnung ist auch im alläglichen Sprachgebrauch erst typisch und bewußt verwendet für die Nachwendezeit. $\mathrm{Zu}$ dem Teiltext 2. Tätigkeitsbeschreibung fallen in den neuen Arbeitszeugnissen hauptsächlich Sachbezeichnungen aus dem alten Diskurs auf: Fachorgan; Publikationsorgane; außerunterrichtliche Aufgaben/Veranstaltungen an der Universität; promovierte mit einer Dissertation A; erbrachte den Nachweis der fakultas docendi.

Aber auch Wendungen wie betriebliche Rationalisierungsmaßnahmen; war organisatorisch bei der Durchführung beteiligt; leistete ab; Aus- und Weiterbildung; Zielstellung und Aufgabenstellung; die daraus resultierenden Erfahrungen; kam ihren Verpfichtungen nach entstammen den normierten, intersubjektiven Wissensbeständen dieser Textsorte in der DDR, manifestiert im Textdenken und in Formulierungsgewohnheiten.

Der Teiltext 3. Leistungsbeurteilung wird vorsichtig formuliert. Hohes Verantwortungsbewußtsein, hohes Pfichtbewußtsein, hohe Einsatzbereitschaft sind durch die verwendeten Kollokationen markiert, im Sinne eines vorgeschlagenen Markiertheitsbegriffes von Anderson (1984, S. 62), entsprechen aber durchaus möglichen Formulierungsangeboten der Ratgeberliteratur, werden jedoch dort nicht in dieser relativen Häufigkeit und in den festen Wortverbindungen wie in den untersuchten VergleichsArbeitszeugnissen verwendet. Der verglichene Umgebungskontext in den Beurteilungen bleibt ebenfalls stereotypisiert, so daß die Relation Sachverhaltsgestaltung und Art der Handlungsdurchführung als "gewohnt" bezeichnet werden kann, wie etwa 
... stelle sich mit großem Engagement und hohem Verantwortungsbewußtsein jeder übertragenen Aufgabe.

... erwarb sich bei der Ausübung dieser Funktion große Verdienste.

Die ihr übertragenen Aufgaben löste sie mit großer Einsatzbereitschaft und hohem Verantwortungsbewußtsein.

Teiltext 4. Führung und Sozialverhalten - beide Lexeme als Schlüsselwörter für den Teiltext sind neu und ersetzen im vertrauten Muster Einstellung zur Gesellschaft, Einstellung zur Arbeit und zum Kollektiv. Deshalb bleibt das Kollektiv auch in fast allen Arbeitszeugnissen als Dublette bestehen:

Arbeitskollektiv, Ärztekollektiv, Stationskollektiv, Lehrerkollektiv, Klassenkollektiv, Schülerkollektiv.

Stereotype Wendungen wie:

machte sie zu einem wertvollen Mitglied des Kollektiv;

leistete einen Beitrag zur Erhöhung des Ansehens;

arbeitete zum Wohle unseres Betriebes;

fügte sich in das Lehrerkollektiv gut ein,

sind typische Kollokationen, die als DDR-markiert gelten können und in den Häufigkeitstabellen in den untersuchten DDR-Beurteilungen einen vorderen Platz einnahmen.

Teiltext 5. Führungsqualitäten und Aufstiegseignung konnten nur in der Umschreibung in ihrer Funktion als Leiterin und einmal, schon angelehnt an neue Denkweisen als ärztliche Laufbahn beschrieben werden. Hier können aber Einschränkungen im vorhandenen Analysematerial begründet sein.

Die für Arbeitszeugnisse in der Ratgeberliteratur vorgeschlagenen Wünsche Teiltext 6. Schluß/Gute Wünsche unterbleiben meist in den hier analysierten neuen Arbeitszeugnissen/Beurteilungen. Als fakultativer Teiltext waren sie in DDR-Beurteilungen in der Regel auch nicht üblich und nicht nötig, da ein Großteil der Beurteilungen zu anderen Zwecken geschrieben wurde als zum Arbeitsplatzwechsel. Falls Wünsche formuliert werden, klingen sie mitunter sehr ungewöhnlich wie in dem folgenden Arbeitszeugnis:

Unabdingbare Struktur- und Rationalisierungsmaßnahmen in unserem Unternehmen zwingen uns dazu, das bestehende Arbeitsverhältnis mit Herrn $X$ zu beenden. Wir bedauern das sehr und hoffen, daß Thre Institution Herrn $X$ eine seinen Fähigkeiten und Kenntnissen entsprechende Arbeitsaufgabe anbieten kann. Wir wünschen Herrn $X$ für weitere berufliche Jahre noch viel Erfolg, Schaffenskraft und Wohlergehen. 
Die Unterschrift, von meist noch mehreren Vorgesetzten, beendet das Arbeitszeugnis, wobei auch die Titel wie Medizinalrat, Diplom-Mediziner, amtierender Direktor, amtierender Abteilungsleiter, Vorstandsvorsitzender oder auch gar keine Berufsbezeichnung DDR-typische Benennungen bzw. Übergangsformen sind.

Wenn man die Sequenzierung als Prinzip der logischen Abfolge der einzelnen Teiltexte betrachtet, die ja als globale Vorgabe in diesem Textmuster kaum zu modifizieren ist, so fält auf, daB, bevor mögliche gute Wünsche zum Schluß formuliert werden, häufig noch eine zusammenfassende Einschätzung gegeben wird, die mit insgesamt, zusammenfassend, abschließend kann eingeschätzt werden beginnt.

Überhaupt wird die logische Abfolge mehrfach durchbrochen, indem besonders nach den Teiltexten 3-5 nochmals auf die Tätigkeitsbeschreibung bezug genommen wird. Das geschieht fast ausschlieflich in den Arbeitszeugnissen/Beurteilungen, die von den neuen Vorgesetzten (Ost) geschrieben werden, während Vergleichs-Arbeitszeugnisse von Personalleitern (West) geschrieben, die Sequenzfolge genau einhalten. Hier kann spekulativ Unkenntnis genauer Musterhierarchien angenommen werden.

\subsection{Adaption neuer normierter Formulierungsmuster}

Es fiel auf, $d a B$ in einzelnen Teiltexten als Implementierung bestimmte Lexeme bevorzugt verwendet werden. Das betriftt Teile der Tätigkeitsbeschreibung, wo eine instabile Mischung von tradierten und neuen Formen vorhanden ist. Dabei kann man davon ausgehen, daß besonders identitätsstiftende und vertrauter werdende Bezeichnungen für die Arbeitsumgebung dazu beitragen.

So werden Benennungen komplementär zu Betrieb gewählt, wie etwa Firma, Unternehmen, Auftraggeber, Unternehmungsführung, MarketingBereich, Management-Bereich.

Das für das Sozialverhalten wichtige Kollektiv im Teiltext 4. wird häufig im gleichen Text auch als Team, (Stationsteam, Ärzteteam) bezeichnet.

Bei der Leistungsbeurteilung wird die vorgegebene standardisierte Ausdrucksweise stets zur vollsten Zufriedenheit fast ausschließlich benutzt. Zielstrebig und engagiert ergänzen die vorgeschlagene Bewertung und gehören zu den typischen Lexemen dieser Textsorte in Ost und West, wenngleich die Wortverbindungen in den untersuchten DDRBeurteilungen vor allem folgende waren: 
politisches Engagement für unseren Staat, weltanschaulich gefestigtes Engagement, fachlich und organisatorisches Engagement, Engagement und Selbstlosigkeit für die Verwirklichung der Parteibeschlüsse.

In den vorliegenden Arbeitszeugnissen aus den alten Bundesländern wird Engagement in folgenden Verbindungen verwendet: reges Engagement; großes berufliches Engagement; Interesse, Fleiß und Engagement, so daß durch die unterschiedlichen Kollokationen Engagement unterschiedlich konnotiert ist, zwar als musterbezeichnender Ausdruck existiert, nun aber für den Rezipienten (Ost) fremd wirkt.

\subsection{Individuelles Formulieren}

Im Zusammenspiel von Musterbefolgen (Leistungsbeurteilung) und Improvisieren, weil das Inventar des Musters im bewuBten Vermeiden von Tradition umgangen werden soll oder nicht so geläufig ist, kommt es zu bewuBter, aber auch ungewollter Kreativität beim Ausfüllen des Musters. Die Möglichkeiten des individuellen Abweichens vom konventionell vorgegebenen Rahmen sind bei dieser Textsorte relativ gering.

Es fällt auf, $\mathrm{daB}$ vorgegebene, fremd empfundene Bewertungsausdrücke wie erfolgsorientiert, innovativ, souverän, Kreativität (Teiltext 3) oder Prestige, kooperativer Führungsstil, Karriere, Autorität, Führungspraxis, Teamfähigkeit, Kommunikationsfähigkeit, Durchsetzungsvermögen (Teiltext 4/5) umgangen werden. Man kann hier von Konnotationen sprechen, die auf konventionellen Gebrauchsrestriktionen beruhen und somit eine stilistische Bedeutung besitzen. Lexeme wie Karriere/Karriereaufstieg gehörten in DDR-Beurteilungen nicht zum Inventar und wurden in alltagssprachlichen Kontexten eher negativ konnotiert, wenn überhaupt gebraucht.

Bei der Realisierung der Teiltexte Führung/Führungsqualitäten werden als Handlungsalternativen individuelle Formulierungen gewählt wie seine Erwägungen waren treffsicher, seine Sensibilität bei der Tätigkeit ist hervorzuheben, ihre diagnostische Strategie (Teiltext 3); aufgeschlossener Gesprächspartner; entwickelt sich zu einer großen Hilfe; gab Denkanstöße zur Veränderung der angetroffenen Realität; erfreut sich hoher Wertschätzung der Kollegen (Teiltext 4/5). 


\subsection{Dominante Teiltextmuster entfallen}

Die soziolinguistische Fragestellung: „Zu welchem Zeitpunkt und in welcher Umgebung ändert sich die sprachliche Struktur aufgrund welcher Verhältnisse?" (Dittmar 1973, S. 157) ist ein zentrales Problem des Sprachwandels und soll hier explizit genannt werden.

Neue Konventionen verlangen andere Muster. Konventionell unverträglich in der neuen sozialen Situation ist der Teiltext Einstellung zur Gesellschaft/Angaben zur politisch-ideologischen Haltung, der prototypisch und zentraler Bestandteil für Beurteilungen war und von stereotypisierten sprachlichen Mitteln dominiert war.

Eine Häufigkeitsstatistik der untersuchten Texte zeigt in Frequenz und Distribution folgende Schlüsselwörter:

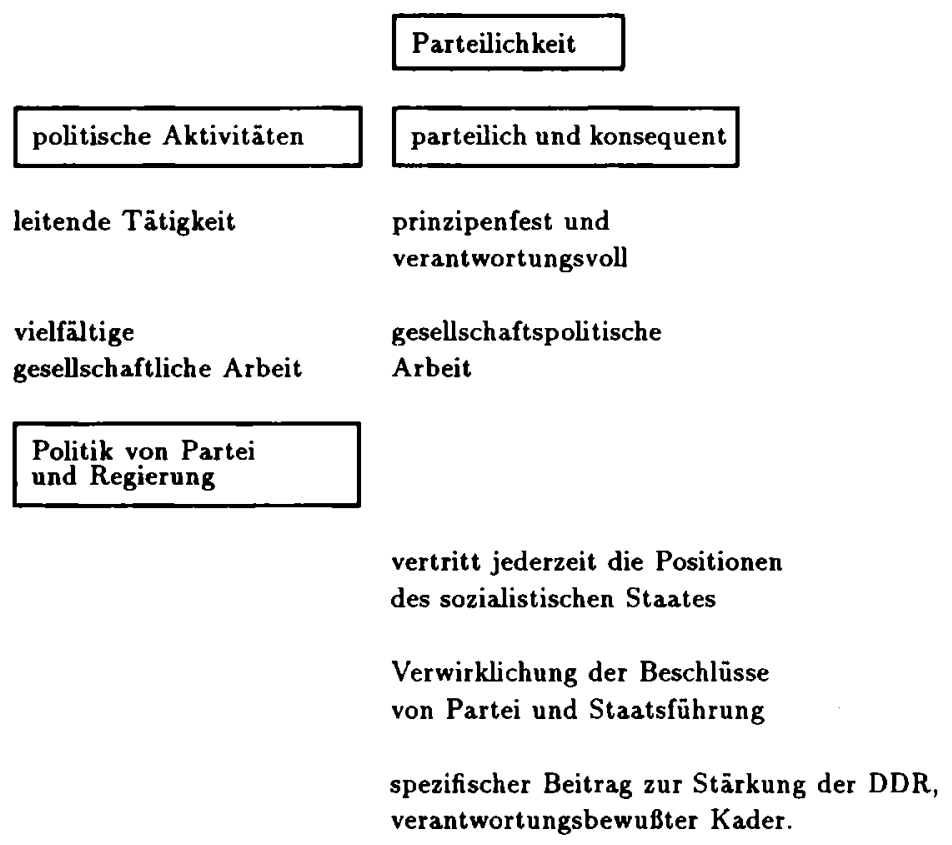

Diese Schlüsselwörter können als prototypische Stilelemente für das Textmuster gelten, die der normativen Vorstellung entsprachen und durch die veränderten normativen Bedingungen im neuen Textmuster entfallen. 


\section{Zusammenfassung}

Anhand der vorliegenden Analysen wird deutlich, daß das Textmuster Arbeitszeugnis unter den situativen Bedingungen modifiziert realisiert wird. Die Veränderungen resultieren aus:

1. Unvollkommene Kenntnis des Textmusters - Ratgeberliteratur ist weitgehend unbekannt/ungewohnt zu benutzen (Anrede, SchluB)

2. BewuBtes Eliminieren tradierter Teiltextmuster (politische Entwicklung, Parteizugehörigkeit)

3. Bewußtes Umgehen fremd empfundener Teiltexte und ihrer vorgegebenen Formulierungsangebote (erfolgsorientiert, Führungspraxis)

4. Fehlende Kompatibilität von Wissensbeständen (Dissertation $A$ )

5. UnbewuBte Verwendung von tradierten Formen in strukturell übereinstimmenden Teiltexten (2/3) (Kollektiv, Aus- und Weiterbildung, außerunterrichtliche Aufgaben)

6. BewuBte Implementierung bzw. Substitution bereits vertraut empfundener neuer synonymer Formulierungsmuster (Firma, Unternehmen).

Als verallgemeinerte Stilwirkung im Vergleich zu Musterarbeitszeugnissen und vorliegenden Arbeitszeugnissen westdeutscher Arbeitgeber erscheinen aus Rezipientensicht die im Musterkonflikt entstandenen synthesehaften gemischten Beurteilungen/Arbeitszeugnisse $\mathrm{k} \mathrm{n}$ a p p. Die Art der Durchführung wirkt oft $u n b$ e h o l f e $n$ aber auch f r e m d a r t ig und $n$ e u a $r$ t i g in Bezug auf tradiertes Musterwissen und weggefallene Standardorientierungen. Die Art der Themenbehandlung wird als notwendig zeitgemäß empfunden. Durch das Weglassen der als fakultativ empfohlenen guten Wünsche zum SchluB wirken die Texte un persönlich, steif und distanziert gegenüber Wohlwollen und Herzlichkeit ausstrahlenden SchluBformulierungen in Arbeitszeugnissen, geschrieben von Arbeitgebern aus den alten Bundesländern. Die Stilzuschreibung ,wohlwollend" wird durch Formulierungen wie: Bedauern über Fortgang; allerbesten Dank; Gesundheit, Glück und Erfolg in der fakultativen GruBsequenz gestützt.

Konflikte zwischen Schreibern und Rezipienten von neuen Arbeitszeugnissen resultieren auch aus der Stilwirkung, besonders der Art der Themenbehandlung und der Art der Durchführung, die in Relation zu dem konventionell Erwartbaren gewertet wird. Hier werden auch Unterschiede im momentanen gemeinsamen Wissen zur Realisierung des Textmusters deutlich. Häufig verfügt der Rezipient über die besseren Kenntnisse.

Abschließend sei noch auf einen Konflikt hingewiesen, der sich aus unterschiedlichen Relationen der Adressiertheit ergibt. Herkunftsort, Wohnort, Arbeitsort und zukünftiger Arbeitsort sind für die Interpretation 
von Arbeitszeugnissen in Zukunft mit zu beachten. Konfliktsituationen entstehen - jetzt und in Zukunft -, wenn Produzent und Rezipient durch traditionelle Verhaltensmuster, beispielsweise die Art und Weise, Beurteilungen zu interpretieren (Fix 1993), die kommunikative Andersartigkeit in einem besonders sensiblen persönlichen Bereich verspürten. Das führt dazu, daß Arbeitnehmer der neuen Bundesländer, die Arbeitszeugnisse fordern, weil ihr Arbeitsverhältnis beendet wird, häufig Vergleiche mit Ratgeberliteratur auf Hinweis westdeutscher Bekannter vornehmen und entsprechende Formulierungen fordern, ja sogar mit dem Arbeitsgericht drohen. Dabei werden Auszüge aus Ratgeberliteratur dem Zeugnisschreiber vorgelegt oder es werden Tätigkeitsbeschreibungen vom Arbeitnehmer selbst formuliert und als Anhang an das Arbeitszeugnis mit Unterschrift vom zuständigen Vorgesetzten angefügt. Um solchen Konfliktsituationen vorzubeugen, haben beispielsweise Lehrer in SachsenAnhalt für das Schreiben von Schülerbeurteilungen und Schullaufbahnempfehlungen Formulierungshilfen von Niedersachsen (Lüneburg) zur Verfügung gestellt bekommen.

Hier sollte an einer Textsorte aus dem Textsortenspektrum der Alltagswelt die Konfliktsituation aufgrund der Divergenz unterschiedlicher Kommunikationskulturen am Fortwirken von kommunikativen Mustern sowie am beginnenden Transfer zum Ausgleich von Differenzen gezeigt werden, was für einen längeren Zeitraum noch, auch bei dieser stark normierten Textsorte, zu einer regionaltypischen Spezifik sprachlicher Ausfüllung des Textmusters Arbeitszeugnis führt, wie das abschließende Beispiel eines Arbeitszeugnisses nach neunjähriger Tätigkeit zeigen soll:

\begin{abstract}
Abschlußbeurteilung
Frau $X$, geb. am 24.03.41, arbeitete vom 1.11.1982 bis zum 31.12.1991 als Sachbearbeiter in der Vetriebsabteilung der Melany-Konfektionsund Handels AG, ehemals VEB Kindermoden. Ihr Aufgabenbereich beinhaltete den ÁbschluB von Vertrāgen zu Messen, Auswertung der Vertragsabschlüsse, eine ordnungsgemāße Durchführung von monatlichen Inventuren sowie eine tägliche Überwachung der termin- und qualitätsgerechten Einhaltung der Lieferverträge und weitere Verkäufe und Angebote zu tätigen. Diese ihr übertragenen Aufgaben löste sie stets zur vollen Zufriedenheit. Fleiß und Verantwortungsbewußtsein gehörte zu ihrer tāglichen Arbeit. Auf Grund von Rationalisierungsmaßnahmen innerhalb des Unternehmens wurde Frau X die Kündigung zum 31.12.1991 ausgesprochen.
\end{abstract}

Vorstandsvorsitzender

Ziel solcher Analysen alltagssprachlicher Textsorten ist es, entstehende und bereits entstandene Mischformen differenziert zu beschreiben, Wissensdifferenzen an Einzelbeispielen aufzuzeigen und Akzeptanz für durch Diffusion entstehende Musterveränderungen zu erwirken. 


\section{Literatur}

Andersson, Sven-Gunnar (1984): Wortwanderung. Zur Beschreibung der deutsch-deutschen Sprachsituation im Bereich des Wortschatzes. In: Deutsche Sprache 12, S. 54-84.

Bauer, Dirk (1990): Zwei deutsche Staaten! - Zwei deutsche Sprachen? Überlegungen zur Entwicklung der germanistischen Forschung und zum Verhältnis von Sprachwissenschaft und Politik. In: Deutsche Sprache 18, S. 218240.

Becker-Mrotzek, Michael (1990): Kommunikation und Sprache in Institutionen. Ein Forschungsbericht zur Analyse institutioneller Kommunikation. In: Deutsche Sprache 18, S. 158-190, S. 241-259.

Bellmann, Günter (1991): Wandlungen im Wortschatz der deutschen Gegenwartssprache: Der Wortschatz und die deutsche Einheit. In: Beiträge zur deutschen Linguistik, Methodik und Didaktik 5. Kyoto 1991, S. 21-32.

Brinker, Klaus (1985): Linguistische Textanalyse. Berlin.

Brinkmann, Bettina/Buder, Andreas/Dawin, Andrea/Osburg, Anke (1992): Ein Staat - eine Sprache? Empirische Untersuchungen zum englischen EinfluB auf die Allgemein-, Werbe- und Wirtschaftssprache im Osten und Westen Deutschlands vor und nach der Wende. Frankfurt.

Burkhardt, Armin/Fritzsche, Klaus Peter (Hg.) (1992): Sprache im Umbruch. Politischer Sprachwandel im Zeichen von "Wende” und „Vereinigung”. Berlin/New York.

Dimter, Matthias (1981): Textklassenkonzepte heutiger Alltagssprache. Kommunikationssituation, Textfunktion und Textinhalt als Kategorien alltagssprachlicher Textklassifikation. Tübingen. (= Reihe germanistische Linguistik, 32).

Dittrich, Helmut (1988): Arbeitszeugnisse schreiben und verstehen. München.

Ehlich, Konrad (1986): Die Entwicklung von Kommunikationstypologien und die Formbestimmtheit sprachlichen Handelns. In: Kallmeyer, Werner (Hg.): Kommunikationstypologie. Handlungsmuster, Textsorten, Situationstypen. Jahrbuch 1985 des Instituts für Deutsche Sprache. Düsseldorf. S. 47-72.

Erlebach/Hoft/Ihlefeld/Zehner (1983): Schülerbeurteilung. 10. Aufl.. Berlin.

Fix, Ulla (1992): Stil als komplexes Zeichen im Wandel. In: Zeitschrift für germanistische Linguistik 20.2, S. 193-209.

Fix, Ulla (1994): Texte mit doppeltem Boden. Erscheint: Wien 1994.

Fleischer, Wolfgang (1987), (Autorenkollektiv): Wortschatz der deutschen Sprache in der DDR. Leipzig.

Franke, Wilhelm (1987): Texttypen - Textsorten - Textexemplare. Ein Ansatz zu ihrer Klassifizierung und Beschreibung. In: Zeitschrift für germanistische Linguistik 15, S. 263-281.

Franke, Wilhelm (1991): Linguistische Texttypologie. In: Brinker, Klaus (Hg.): Aspekte der Textlinguistik. Tübingen. S. 157-182. (= Reihe Germanistische Linguistik, 106/107). 
Gläser, Rosemarie (1990): Fachtextsorten im Englischen. Tübingen.

Good, Colin (1993): Über die „Kultur der Mißverstāndnisse” im vereinten Deutschland. In: Muttersprache 103, 3, S. 249-259.

GroBe, Ernst Ulrich (1976): Text und Kommunikation. Stuttgart.

Gülich, Elisabeth/Raible, Wolfgang (Hg.) (1972): Textsorten. Differenzierungskriterien aus linguistischer Sicht. Frankfurt. 2. Aufl., Wiesbaden 1975 .

Gülich, Elisabeth (1985): Textsorten in der Kommunikationspraxis. In: Kallmeyer, Werner (Hg.): Kommunikationstypologie. Jahrbuch 1985 des Instituts für Deutsche Sprache. Düsseldorf 1986. S. 15-46. (= Sprache der Gegenwart, Bd. 67).

Hannappel, Hans/Melenk, Hartmut (1984): Alltagssprache. München.

Hellmann, Manfred W. (1990): DDR - Sprachgebrauch nach der Wende - eine erste Bestandsaufnahme. In: Muttersprache 100, S. 266-286. Dazu Diskussion: ebd. 101, S. 66-70.

Kießling, Kurt (Hg.) (1969): Ausdruckslehre. 7. Aufl. Leipzig.

Kühn, Ingrid (1992): Straßennamen nach der Wende. In: Namenkundliche Informationen. Leipzig.

Kūhn, Ingrid (1994): Sprachberatung in den neuen Bundesländern - Hilfe bei deutsch-deutschen Sprachproblemen. In: Muttersprache 104, Heft 2.

Lerchner, Gotthard (Hg.) (1992): Sprachgebrauch im Wandel. Anmerkungen zur Kommunikationskultur in der DDR vor und nach der Wende. Frankfurt/M. et al. (= Leipziger Arbeiten zur Sprach- und Kommunikationsgeschichte, Bd. 1).

Lerchner, Gotthard (1992): Broiler, Plast(e) und Datsche machen noch nicht den Unterschied. Fremdheit und Toleranz in einer plurizentrischen deutschen Kommunikationskultur. In: Lerchner (Hg.) (1992), S. 297-332.

Liebsch, Helmut (Hg.) (1976): Deutsche Sprache. Leipzig.

Lux, Peter (1993): Ostdeutsch-Westdeutsch: Zwei Sprachen. In: Leipziger Volkszeitung. 31.12.1993.

Möhn, Dieter/Pelka, Roland (1984): Fachsprachen. Tübingen.

Nasemann, Andrea (1992): Arbeitszeugnisse durchschauen und interpretieren. Niedernhausen.

Oschlies, Wolf (1989): Würgende und wirkende Wörter. Deutschsprechen in der DDR. Berlin.

Pātzold, Jörg (1992): Zwischen Indirektheit und Sprachlosigkeit. Der Umgang der Presse in der DDR zwischen dem Stern-Interview Hagers und Oktober 1989 mit der Wirklichkeit des Real Existierenden. In: Welke u.a. (Hg.) (1992), S. 93-110.

Petersen, Jens (1986): Sprache in der gesellschaftlichen Öffentlichkeit. Frankfurt.

von Polenz, Peter (1988): „Binnendeutsch” oder plurizentrische Sprachkultur? Ein Plädoyer für Normalisierung in der Frage der "nationalen" Varietāten. In: ZGL 16, S. 198-218. 
von Polenz, Peter (1993): Die Sprachrevolte in der DDR. Im Druck: Germanistische Linguistik.

Presch, Gunter/Gloy, Klaus (Hg.) (1976): Sprachnormen II. Stuttgart-Bad Cannstatt 1976.

Presch, Gunter (1991): Arbeitszeugnisse. Informationsbroschüre des Germanistischen Seminars der Universitāt Hamburg. Hamburg.

Püschel, Ulrich (1982): Die Bedeutung von Textsortenstilen. In: Zeitschrift für germanistische Linguistik 10, S. 28-37.

Reséndiz, Julia Liebe (1992): Woran erkennen sich Ost- und Westdeutsche? Eine Spracheinstellungsstudie am Beispiel von Rundfunksendungen. In: Welke u.a. (Hg.) (1992), S. 127-140.

Rolf, Eckhard (1993): Die Funktion der Gebrauchstextsorten. Berlin.

Ruge, Elisabeth (1993): Behördenkorrespondenz. Niedernhausen.

Sandig, Barbara (1987): Textwissen. Beschreibungsmōglichkeiten und Realisierungen von Textmustern am Beispiel der Richtigstellung. In: Engelkamp/Lorenz/Sandig (Hg.): Wissensrepräsentation. St. Ingbert. S. 115155.

Sandig, Barbara (1989): Stilistische Mustermischung in der Gebrauchssprache. In: Zeitschrift für Germanistik 2/1989, S. 133-150.

Schrōder, Marianne (1992): Lexikographische Nach-Wende - Ein Überarbeitungsbericht. In: Lerchner (Hg.) (1992), S. 263-296.

Schmidt, Wilhelm (1981): Funktional-kommunikative Sprachbeschreibung. Leipzig.

Welke, Klaus/Sauer, Wolfgang W./Glück, Helmut (Hg.) (1992): Die deutsche Sprache nach der Wende. Hildesheim usw. (= Germanistische Linguistik 110-111/1992).

Wittgenstein, Ludwig (1953): Philosophische Untersuchungen. Frankfurt.

Wypijeski, Wolfgang (1992): 99 Zeugnisse. München.

Wörterbuch der deutschen Gegenwartssprache, hrsg. v. Klappenbach, Ruth/ Steinitz, Wolfgang. Berlin 1978.

Duden, Deutsches Universalwörterbuch, hrsg. v. Drosdowski, Gūnter. Mannheim 1989.

Rückläufiges Wörterbuch der deutschen Gegenwartssprache, hrsg. v. Mater, Erich, Leipzig 1967.

Synonymwörterbuch, hrsg. v. Görner, Herbert/Kempcke, Günter. Leipzig 1973. 